\title{
TOUSCHEK EFFECT IN APPROXIMATION OF TWO DIMENSIONAL COLLISIONS
}

\author{
D.Yu. Golubenko, S.A. Nikitin* \\ BINP SB RAS, Novosibirsk, 630090, Russia
}

\begin{abstract}
An approach to account for the two-dimensional character of collisions of particles inside a relativistic electron bunch and to estimate influence of this factor upon parameters of a beam in a synchrotron is developed.
\end{abstract}

\section{INTRODUCTION}

Processes of mutual scattering of particles inside a relativistic bunch, i.e. the Touschek effect, define to large extent the sizes and lifetime of electron and positron beams in storage rings and colliders at rather low energy. A well-known theory for this effect stated consistently in [1] bases on a one-dimensional approximation of two-particle Coulomb interaction. Such approach is practically true for conventional colliders with flat orbits, where the horizontal equilibrium size of the beam exceeds significantly the vertical one. Further development of methods of control over the vertical size with the help of longitudinal and skewquadrupole fields and particularly appearance of the round beam conception [2], can make the mentioned approximation seem insufficient. The presented work aims at developing an approach to account for the two-dimensional character of particle interaction inside a bunch and estimate its influence upon beam parameters [3].

\section{DISTRIBUTION FUNCTION}

\subsection{Main definitions}

Let list some qualitative considerations clarifying the main difference in description of the processes of intra-bunch particle scattering in a two-dimensional electron beam as compared with a conventional one-dimensional approximation (flat beam). Let designate $p=\left|\overrightarrow{p_{1}}-\overrightarrow{p_{2}}\right| / 2$ the particle momentum in the center-of-mass system (c.m system) moving together with the beam. Generally momenta of the colliding particles $\overrightarrow{p_{1}}$ and $\overrightarrow{p_{2}}$ have both horizontal $\left(p_{X}\right)$ and vertical $\left(p_{Z}\right)$ components. Similarly to the kinetic gas theory, variance of the momentum $p$ is doubled as compared with the one-dimensional case, if the efficient temperatures by degrees of freedom are equal $\left(\sigma_{p_{X}}^{2}=\sigma_{p_{Z}}^{2}=\sigma_{p}^{2}\right)$ : $<p^{2}>=\sigma_{p}^{2}$. Here temperature for either of the directions of motion is defined by the spread of the corresponding component of the transverse particle momentum, equal for the laboratory (lab) and accompanying (c.m.) systems. Variance growth means a change of the form of the distribution function as to the momentum $p$ and, therefore, regarding the relative velocity of the colliding particles $\nu=2 p / m$

\footnotetext{
*nikitins@inp.nsk.su
}

( $m$ is the electron mass). That must influence the efficiency of the intra-bunch scattering processes as a whole, since the Møller differential cross-section is inversely proportional to the forth degree of $\nu$.

It is important most of all to obtain the form of the distribution function $f(p)$ that would in a certain way take into account the two-dimensional character of two-particle interaction inside an electron bunch under the condition that the vertical temperature $\sigma_{p_{Z}}^{2}$ is not small. Note that only in one case, namely when the $X$ - and $Z$-temperatures are equal, the function $f(p)$ takes the form of two-dimensional Maxwell distribution: $f(p) \propto p \cdot \exp \left(-p^{2} / \sigma_{p}^{2}\right)$.

\subsection{One-dimensional case (flat beam)}

Let the flat electron beam in the synchrotron be characterized by the Gauss distribution of the trajectory angles in the horizontal plane $\left(X^{\prime}\right)$ with the dispersion $\sigma_{X^{\prime}}$. We will express the momentum in the c.m. system as $p=m c \gamma \xi=$ $p_{0} \gamma \xi$ and the spread of the transverse momentum in the beam as $\sigma_{p}=p_{0} \gamma \sigma_{X^{\prime}}$, where $\gamma$ is the relativistic factor and $\xi=\left|X_{1}^{\prime}-X_{2}^{\prime}\right| / 2=\nu / 2 c$ is the relative velocity of colliding particles (" 1 " and " 2 ") in units of the velocity of light. The distribution function $f(p)$ for the flat beam is given by the expression [1]

$$
f(p) d p=\frac{2}{\sqrt{\pi} \sigma_{p}} \exp \left(-\frac{p^{2}}{\sigma_{p}^{2}}\right) d p .
$$

\subsection{Approximation of two-dimensional colli- sions}

Let find distribution of the squared relative velocity in the electron beam

$$
\nu^{2}=\nu_{X}^{2}+\nu_{Z}^{2}
$$

where the designations of the velocity components, $\nu_{X}$ and $\nu_{Z}$, are introduced, which in turn are defined as the difference between the components of velocities of particles " 1 " and " 2 ". We derive the distribution function, assuming that the values $\nu_{X}^{2}$ and $\nu_{Z}^{2}$ are "statistically" independent.

In the mentioned assumption, the probability density for the value $Y=\nu^{2}$ is obtained in the following form:

$$
\begin{gathered}
f_{Y}=\frac{1}{4 \sigma_{X^{\prime}} \sigma_{Z^{\prime}}} \exp \left[-\frac{Y}{2}\left(\frac{1}{4 \sigma_{X^{\prime}}^{2}}+\frac{1}{4 \sigma_{Z^{\prime}}^{2}}\right)\right] . \\
I_{0}\left[\frac{Y}{2}\left(\frac{1}{4 \sigma_{X^{\prime}}^{2}}-\frac{1}{4 \sigma_{Z^{\prime}}^{2}}\right)\right],
\end{gathered}
$$

$I_{0}(x)$ is the modified Bessel function. Let introduce the parameter $k=\sigma_{X^{\prime}} / \sigma_{Z^{\prime}}$. In the so-called "round" beam 
$k \rightarrow 1$ and in the flat one $k \rightarrow \infty$. The sought function of distribution as to momentum in the center-of-mass system if the parameter $\mathrm{k}$ is used, it has the following form [3]:

$$
\begin{gathered}
f(k, p) d p=\frac{2 k p}{\sigma_{p}^{2}} \cdot S(w, k) d p \\
S(w, k)=\exp \left[-\frac{w}{2}\left(1+k^{2}\right)\right] I_{0}\left[\frac{w}{2}\left(1-k^{2}\right)\right],
\end{gathered}
$$

where $w=p^{2} / \sigma_{p}^{2}$. Fig. 1 presents the plotted distribution function for different $k$. One can see that at $k \rightarrow \infty$

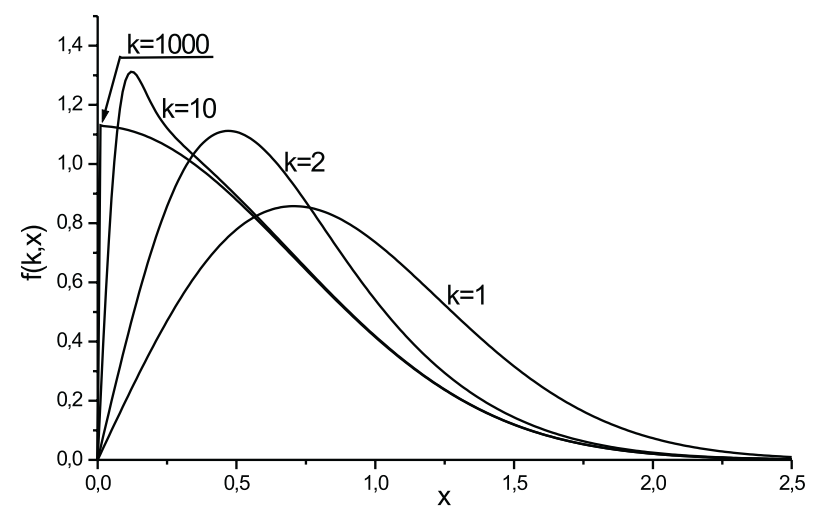

Figure 1: Distribution function $f(k, x), x=p / \sigma_{p}$.

the function approaches the form corresponding to the onedimensional case. At $k \rightarrow 1$ the distribution becomes the two-dimensional Maxwell one.

\section{MODIFIED RATE OF DIFFUSION}

Applying the distribution function of two-dimensional approximation, we obtain the modified rate of energy diffusion due to multiple inner scattering, averaged over the ensemble of beam particles in the laboratory system:

$$
{\overline{\left\{w<\delta E^{2}>\right\}_{l a b}}}=\frac{4 \sqrt{\pi} r_{0}^{2} m^{3} c^{4}}{\sigma_{p}} \cdot B\left(k, \chi_{m}\right) .
$$

Here $w$ is the number of scattering acts per time unit; $<\delta E^{2}>$ - the increment of squared energy averaged over angles; $r_{0}$ - the classical electron radius; $\chi_{m}=p_{m} / \sigma_{p}$; $p_{m}=p_{0} \sqrt{r_{0} / b_{\max }}$ - the classical lower limit of momentum transfer; $b_{\max }$ - the maximal scale of the impact parameter. A novelty is the form of the function $B$, which now depends not only on the parameter $\chi_{m}$ as in the conventional theory [1] but also on the coupling parameter $k$ :

$$
B\left(k, \chi_{m}\right)=\sqrt{\pi} k \int_{\chi_{m}}^{\infty} \sqrt{\frac{1}{\chi}} \cdot \ln \left(\frac{\chi}{\chi_{m}}\right) \cdot S(\chi, k) d \chi .
$$

The function $B\left(k, \chi_{m}\right)$ was constructed in Fig. 2 for different $k$.

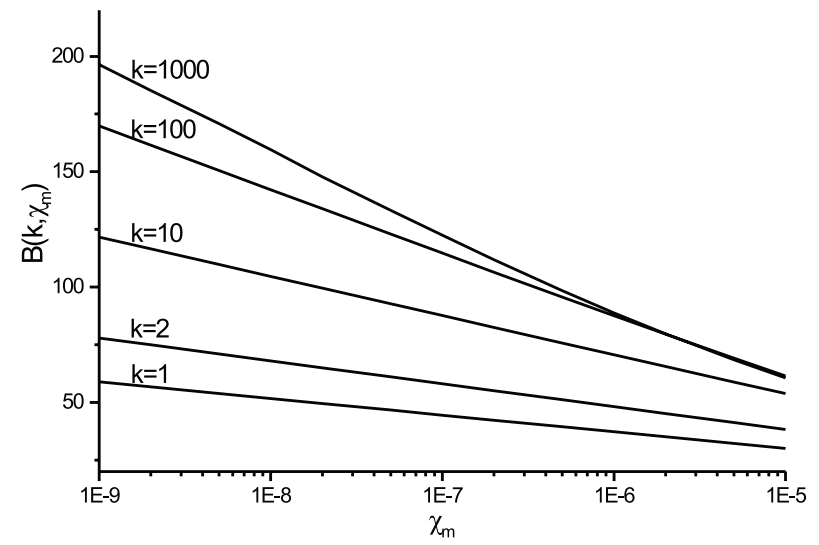

Figure 2: The modified "Touschek function" describing the dependence of the diffusion rate on the parameter $\chi_{m}$.

\section{MODIFIED "LOSS FUNCTION"}

The efficient volume in which a probe particle experiences per time unit a scattering over a relatively large angle and leaves the accelerator is equal to the product of the relative velocity $\nu$ and the loss cross section $\sigma_{\Pi}[1]$ :

$$
\overline{\nu \sigma_{\Pi}}=\int_{A_{p} / \gamma}^{\infty} \nu \sigma_{\Pi} f(k, p) d p=\frac{\sqrt{\pi} r_{0}^{2} m^{3} c^{4}}{\sigma_{p}^{3} \varepsilon} C(k, \varepsilon),
$$

where the "loss function" $C$ depends on the parameters $k$ and $\varepsilon=A_{p} / \gamma \sigma_{p}$ with $A_{p}$ being the "aperture" in the deviation of the longitudinal momentum from the equilibrium value and has the following form

$$
C=\sqrt{\pi} k \varepsilon \int_{\varepsilon}^{\infty} \chi^{-\frac{3}{2}}\left[\frac{\chi}{\varepsilon}-\frac{1}{2} \ln \frac{\chi}{\varepsilon}-1\right] \cdot S(\chi, k) d \chi .
$$

One can see from Fig. 3 that for $\varepsilon<10^{-2}\left(\varepsilon \sim 10^{-4}\right.$ for VEPP-4M) and small $k$ the value $C$ becomes less than in the one-dimensional limit.

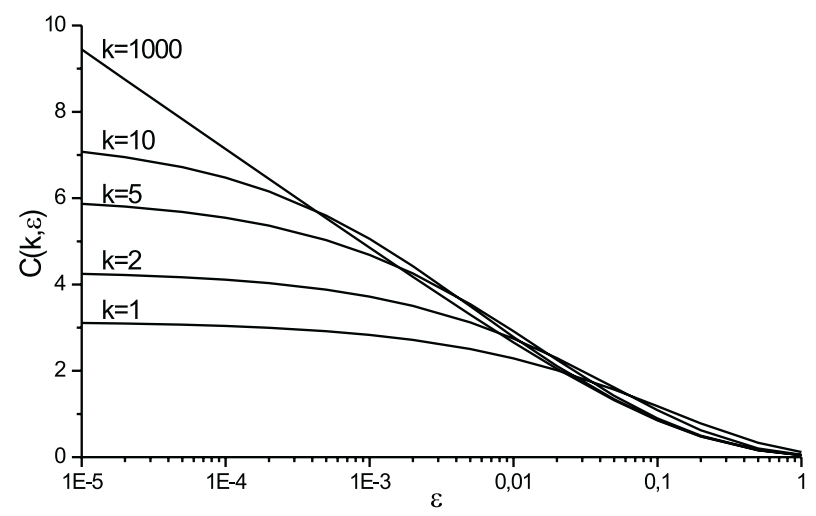

Figure 3: The modified "loss function".

Nevertheless, the definitive result on the beam lifetime depends on other parameters, too. In particular, it depends on $\sigma_{p}$, which also varies with parameter $k$. 


\section{SMOOTH APPROXIMATION}

The determinative process in the integrate Touschek effect is the energy diffusion. Losses of particles (lifetime) at a single scattering depend on the steady dimensions of the beam and are in this sense a secondary process. Coupling of oscillations determines the ratio between the transverse sizes, in particular, the parameter $k$ as well as the tilt of beam cross section. Note that their computation can be carried out with the application of radiation processes only.

To find out main particularities of the two-dimensional approach, we will assume that the indicated geometry of beam is set and, for example, may be described in terms of the theory of difference coupling resonance. Besides, we use a smooth approximation for amplitude functions $\beta_{X}, \beta_{Z}$ of the synchrotron: $\beta_{X, Z}=R / Q_{X, Z}, Q_{X, Z}-$ betatron tunes, $R$ - the average radius. Let denote $u=$ $\left(\sigma_{\gamma} / \gamma\right)^{2}=u_{r}+u_{t}$ - the squared relative energy dispersion; $v=\mathcal{E}_{X}$ - the radial phase volume; $\mathfrak{x}=\mathcal{E}_{Z} / \mathcal{E}_{X}$ the conventional definition of the coefficient of coupling as the ratio of vertical and radial phase volumes, $k=$ $\sqrt{\beta_{Z} /\left(\mathfrak{x} \beta_{X}\right)} ; \mathcal{H}=R / Q_{X}^{3}=$ const $; v_{r} / u_{r}=v_{t} / u_{t}=$ $\mathcal{G}=$ const $; \sigma_{S}=R \alpha \sqrt{u} / Q_{S}$ - the longitudinal beam size, $Q_{S}$ - the synchrotron tune, $\alpha$ - the orbit compression factor. Here the indexes $r$ and $t$ mark the contribution respectively of radiation and Touschek effect. In the smooth approximation, the beam energy spread can be determined from the equation which is similar in the form to that in [1]:

$$
\begin{aligned}
u^{3} & =\frac{\mathcal{N} \tau_{\gamma} r_{0}^{2} c Q_{S} k \sqrt{\beta_{X}} B\left(k, \chi_{m}\right)}{32 \pi \gamma^{3} R \alpha \beta_{Z} \sqrt{\mathcal{G}}(\mathcal{G}+\mathcal{H})}+u_{r} u^{2} . \\
\chi_{m} & =\left(\frac{\mathcal{N}}{8 \pi^{3 / 2} \gamma^{7} \sigma_{X} \sigma_{Z} \sigma_{S}}\right)^{1 / 3} \cdot \frac{r_{0} \beta_{X}}{u(\mathcal{G}+\mathcal{H})} .
\end{aligned}
$$

Here $N$ is the number of particles in the beam; $\sigma_{X} \sigma_{Z}=$ $u \beta_{Z} \mathcal{G} / k$ is the product of transverse beam sizes. The inverse beam lifetime is found from

$$
\frac{1}{\tau}=\frac{\mathcal{N} r_{0}^{2} m^{3} c^{5} Q_{S} k \sqrt{\beta_{X}}}{8 \pi R \alpha \beta_{Z} \mathcal{G}^{3 / 2} A_{p}^{2} u^{2}} \cdot C(k, \varepsilon) .
$$

\section{NUMERIC EXAMPLES FOR VEPP-4M}

Fig. 4 presents the computational results (the curves) on the beam energy spread for the VEPP-4M collider as a function of the particle energy at different values of the coupling parameter $k$. For the sake of comparison, Fig. 5 presents also the experimental results (the dots) [4]. The best compliance with the experiment is reached at $k=4$, which corresponded to the observed ratio of phase volumes. Fig.5 illustrates the difference in the results of computation of $\sigma_{E} / E$ for VEPP-4M in the one-dimensional (the dotted lines) and two-dimensional (the solid lines) approximations. So, in case of the "round beam" $(k=1)$ the correction in the beam energy spread and sizes is $20 \%$. The lifetime is practically the same because of the simultaneous change of the sizes and "loss function".

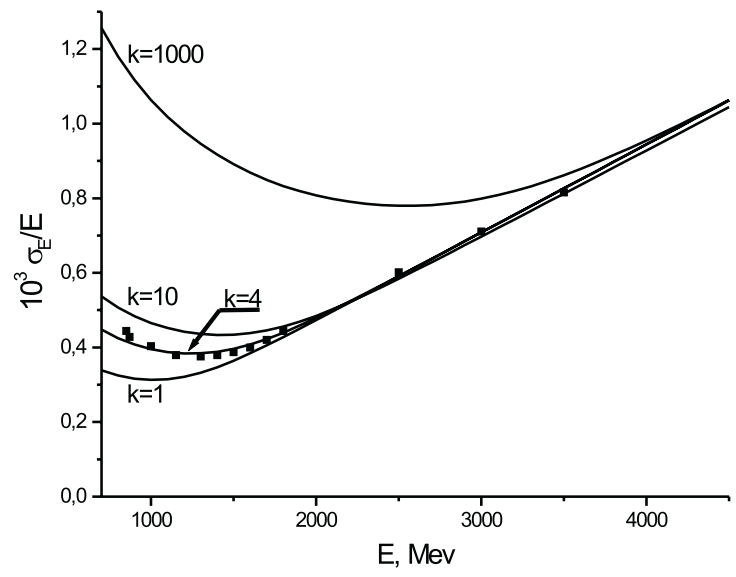

Figure 4: Computed and experimental dependencies of the energy spread on the particle energy in VEPP-4M.

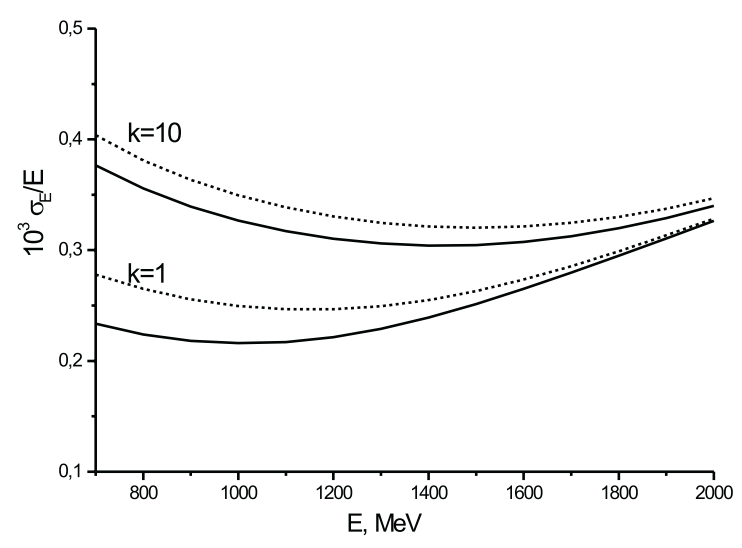

Figure 5: The comparison of two approximations.

\section{CONCLUSION}

Taking into account the two-dimensional character of interaction of particles in the Touschek effect, we found a modified form of the function of distribution as to momentum, diffusion rate and loss rate. We constructed universal characteristic functions describing the effect at different values of the coupling parameter and grading into known dependencies in the flat beam limit. It was numerically shown by the example of VEPP-4M that the correction to the onedimensional theory may be sensible in the beam energy spread and sizes.

\section{REFERENCES}

[1] G.Brook, Cyclic accelerators of charged particles, Moscow. Atomizdat, 1970 (in Russian).

[2] L.M.Barkov et al., Proc. of 14th Int. Conf. on High-Energy Accel., Tsukuba (Japan), p.1385 (1989).

[3] D.Yu. Golubenko and S.A. Nikitin, Preprint BINP 99-110, (1999).

[4] V.A. Kiselev, S.A. Nikitin and I.Ya. Protopopov, Proc.XVII Int. Conf. on High Energy Accelerators (Dubna, Russia, 1998), p.103 (1998). 